\title{
ON THE EXISTENCE OF NON-TANGENTIAL LIMITS OF SUBHARMONIC FUNCTIONS
}

\section{MAYNARD ARSOVE $\dagger$ and ALFRED HUBER}

\section{Introduction}

Suppose that $u$ is a subharmonic function on the open unit disk satisfying the condition

$$
\limsup _{r \rightarrow 1} \int_{0}^{2 \pi}\left|u\left(r e^{i \theta}\right)\right| d \theta<+\infty .
$$

Then, as has been shown by Littlewood $[2 ;$ p. 393], the radial limit

$$
\lim _{r \rightarrow 1} u\left(r e^{i \theta}\right)
$$

will exist finitely for almost all directions $\theta$. If $u$ is harmonic, the limit even exists non-tangentially. That this conclusion is, however, no longer true in the subharmonic case has been pointed out by Zygmund [4; pp. 644-645]; there exists an admitted function $u$ which has no non-tangential limits.

To ensure the existence almost everywhere of non-tangential limits for $u$, a further condition is therefore needed. As such a condition it is natural to consider some restriction on the mass distribution (harmonicity being a rather severe restriction of this type); one might reasonably require that the mass distribution be given by a density function $\lambda$ for which $|\lambda|$ does not grow too rapidly near the boundary. A result of this sort, due to Tolsted [5; pp. 330-332], asserts that non-tangential limits exist almost everywhere if the density $\lambda$ is such that

$$
\int_{0}^{1} \int_{0}^{2 \pi}(1-r)\left|\lambda\left(r e^{i \theta}\right)\right| \log ^{+}\left|\lambda\left(r e^{i \theta}\right)\right| r d \theta d r<+\infty .
$$

Our objective here is to establish the existence almost everywhere of non-tangential limits under some rather simple growth-rate conditions on $|\lambda|$ which are fundamentally different from (1.3). The following two theorems are proved.

Theorem 1. Let $u$ be a subharmonic function on the open unit disk satisfying (1.1), and suppose that the mass distribution for $u$ is given by a density function $\lambda$. If there exist real numbers $M$ and $\delta(<1)$ such that

$$
\left|\lambda\left(r e^{i \theta}\right)\right| \leqslant \frac{M}{(1-r)^{2}}
$$

for $\delta<r<1$ and all $\theta$, then $u$ has finite non-tangential limits at almost all boundary points. The exponent 2 in (1.4) is best possible.

Received 24 July, 1965.

$\dagger$ Research supported by the United States Air Force Office of Scientific Research under Grant AF-AFOSR-936-65. 
Theorem 2. Let $u$ be a subharmonic function on the open unit disk satisfying (1.1), and suppose that the mass distribution for $u$ is given by a density function $\lambda$. If there exist real numbers $p>1$ and $\delta(<1)$ such that

$$
\int_{\delta}^{1} \int_{0}^{2 \pi}(1-r)^{2 p-1}\left|\lambda\left(r e^{i \theta}\right)\right|^{p} r d \theta d r<+\infty
$$

then $u$ has finite non-tangential limits at almost all boundary points. The condition $p>1$ here cannot be replaced by $p \geqslant 1$.

\section{Preliminaries}

In all of the above situations the role of $(1.1)$ is merely to shift the burden of proof from $u$ to a corresponding Green's potential $U$. That is, by virtue of (1.1) $u$ can be expressed as

$$
u=U+h,
$$

where $U$ is the Green's potential of the mass distribution for $u$ and $h$ is a harmonic function satisfying the condition on $u$ in (1.1). Since this condition forces $h$ to have finite non-tangential limits at almost all boundary points, the problem reduces to that of determining when $U$ will admit such limits.

The latter problem was attacked by Tolsted in [5] by obtaining some rather delicate estimates for both the Green's function and the mass density. Our procedure is quite different. It evolves from a technique used by Privalov [3; pp. 31-32] to infer the existence of non-tangential limits of analytic functions as a consequence of the existence of radial limits. Although this technique, based on Vitali's theorem, breaks down even in the case of harmonic functions, certain refinements permit its application to Green's potentials. These involve the normal family theory developed in [1] and the extension of Littlewood's theorem by Tolsted [4] to the effect that at almost all boundary points $\zeta$ the Green's potential $U$ has limit 0 along almost all segments terminating in $\zeta$.

The following notational conventions will be adopted. We use $a$ for 2-dimensional Lebesgue measure, $N_{r}(z)$ for the open disk of radius $r$ about $z$, and $C_{r}(z)$ for the circumference of this disk. The unit disk thus appears as $N_{1}(0)$, and we denote by $R_{\zeta}$ the radius of this disk which terminates in a given point $\zeta$ of $C_{1}(0)$. At any point $\zeta$ of $C_{1}(0)$ the Stolz domain $S_{\alpha}(\zeta)(0<\alpha<\pi / 2)$ will be defined as the circular sector in $N_{1}(0)$ having vertex at $\zeta$, axis along $R_{\zeta}$, vertex angle $2 \alpha$ and radius $\rho_{\alpha}$ equal to half the length of each of the chords of $C_{1}(0)$ lying on the sides of the sector.

In studying the Green's potential $U$ it will be convenient to deal with positive mass distributions, and we shall therefore take the given function as superharmonic, rather than subharmonic. Our derivations centre about four fundamental propositions, which we now list.

Proposimion A. (Tolsted). If $U$ is the Green's potential of a positive mass distribution on $N_{1}(0)$, then for almost all points $\zeta$ on $C_{1}(0)$ the relation 
$U(z) \rightarrow 0$ holds as $z \rightarrow \zeta$ along almost all segments in $N_{1}(0)$ terminating in $\zeta$. (See $[4 ;$ p. 644].)

Proposition B. (Tolsted-Zygmund). If $m$ is a positive mass distribution on $N_{1}(0)$ satisfying

$$
\int_{N_{1}(0)}(1-|z|) d m(z)<+\infty
$$

then for almost all $\zeta$ on $C_{1}(0)$ the mass $m\left[S_{\alpha}(\zeta)\right]$ is finite for each $\alpha(0<\alpha<\pi / 2)$. (See $[5 ;$ p. 325]; the fact that $\alpha$ can be chosen after $\zeta$ follows by considering a sequence of $\alpha$ 's increasing to $\pi / 2$.)

Proposition C. (Arsove). For each $n(=1,2, \ldots)$ let $u_{n}$ be a superharmonic function on a region $\Omega$ with mass distribution given by a density function $\lambda_{n}$. If $\left\{u_{n}\right\}$ is bounded in $L^{1}$ over $\Omega$ and $\left\{\lambda_{n}\right\}$ is bounded in $L^{p}$ over $\Omega$ for some $p>1$, then $\left\{u_{n}\right\}$ is a normal family of continuous functions. If, further, $u_{n} \rightarrow 0$ on a set dense in $\Omega$, then $u_{n} \rightarrow 0$ uniformly on compact subsets of $\Omega$. (See [1]; the final assertion here is an evident consequence of normality.)

Proposition D. Let $U$ be the Green's potential of a positive mass distribution on $N_{1}(0)$, and suppose that $U$ is bounded above on a radius $R_{\xi}$. Then there exists a real number $K$ such that

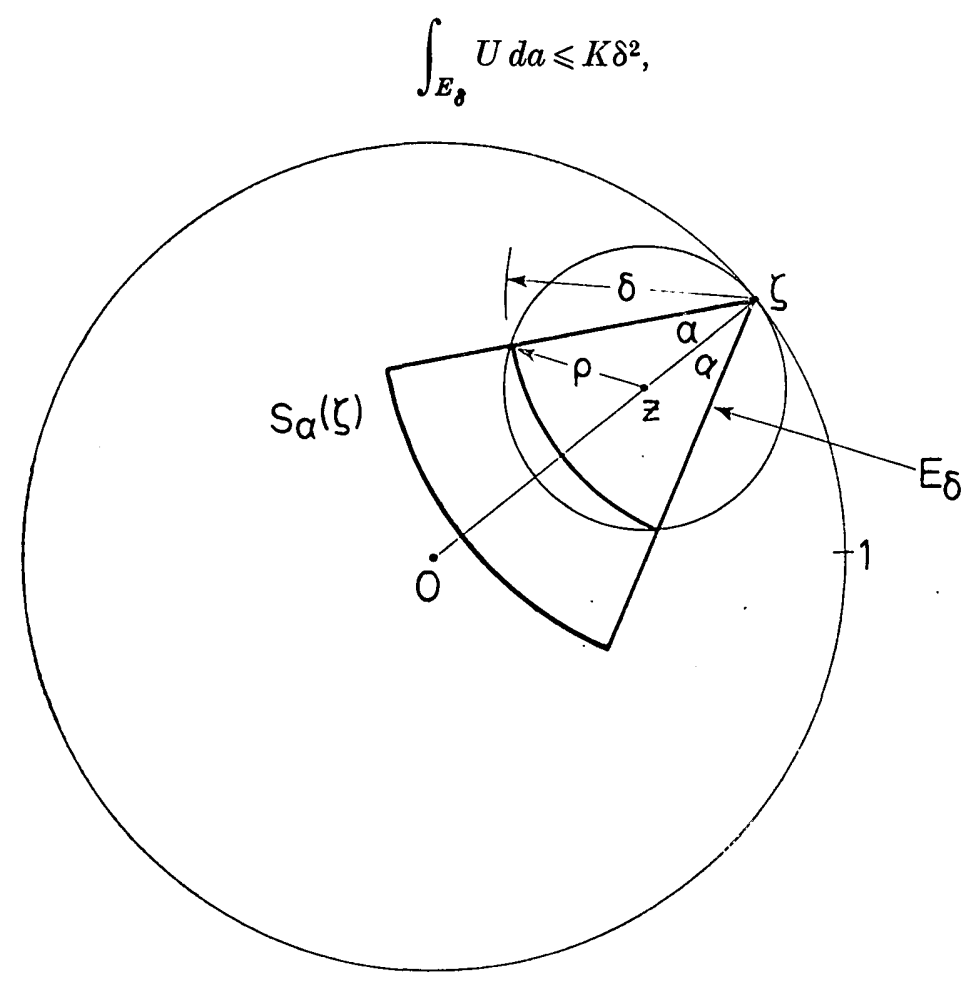

Fig. 1 
where $E_{\delta}$ is defined for $0<\delta<p_{\alpha}$ as the subsector of $S_{\alpha}(\zeta)$ of radius $\delta$ given by $E_{\delta}=S_{\alpha}(\zeta) \cap N_{\delta}(\zeta)$.

Proposition $\mathrm{D}$ is easily deduced from the super mean-value property, and we indicate the proof with reference to Fig. 1. Here a circle $C_{\rho}(z)$ is taken internally tangent to $C_{1}(0)$ at $\zeta$, the radius $\rho$ being so chosen that $C_{\rho}(z)$ passes through the three vertices of the sector $E_{\delta}$. Thus, $\rho=\delta /(2 \cos \alpha)$. By the areal super mean-value property for $U$ over $N_{\rho}(z)$ we obtain

$$
\int_{E_{\delta}} U d a \leqslant \int_{N_{\rho}^{(z)}} U d a \leqslant \pi \rho^{2} U(z) \leqslant \frac{\pi U(z)}{4 \cos ^{2} \alpha} \delta^{2} .
$$

The proposition then follows from the boundedness of $U$ on $R_{\zeta}$.

Proceeding now to the proofs of Theorems 1 and 2, we first establish Theorem 2 and then apply this result in proving Theorem 1.

\section{Proof of Theorem 2}

As has been observed, it suffices to replace $u$ by the Green's potential $U$ of a positive mass distribution with density $\lambda$ satisfying (1.5) for some $p>1$. There is no loss of generality in also taking $\delta=0$, and we shall then show that $U$ has non-tangential limit 0 at almost all points of $C_{1}(0)$.

Before embarking on the proof, however, we remark that the above conclusion is not ensured by (1.5) in the case of $p=1$. Indeed, when $p=1,(1.5)$ reduces to

$$
\int_{0}^{1} \int_{0}^{2 \pi}(1-r)\left|\lambda\left(r e^{i \theta}\right)\right| r d \theta d r<+\infty
$$

and this condition is known to be necessary and sufficient for the mass distribution with density $\lambda$ to admit a Green's potential. On the other hand, an easy modification of the Zygmund example gives rise to a Green's potential admitting no non-tangential limits and having its mass distribution given by a density function.

Returning to the case of $p>1$, we invoke Propositions A and B to infer that for almost all points $\zeta$ of $C_{1}(0)$ and all $\alpha(0<\alpha<\pi / 2)$ the following properties hold :

$U(z) \rightarrow 0$ as $z \rightarrow \zeta$ along $R_{\zeta}$ and along almost all other segments

in $N_{1}(0)$ terminating in $\zeta$, and

$$
\int_{S_{\alpha}(\zeta)}(1-|z|)^{2(p-1)}[\lambda(z)]^{p} d a(z)<+\infty .
$$

Let us fix our attention on one such Stolz domain $S_{\alpha}(\zeta)$ and take $\zeta$ as the origin of polar coordinates $(\rho, \phi)$ as indicated in Fig. 2. Conditions (3.2) and (3.3) then result in

$$
\lim _{\rho \rightarrow 0} U\left(\rho e^{i \phi}\right)=0
$$


for $\phi=0$ and for almost all $\phi$ on $[-\alpha, \alpha]$ and

$$
\int_{0}^{\rho_{\alpha}} \int_{-\alpha}^{\alpha} \rho^{2(p-1)}\left[\lambda\left(\rho e^{i \phi}\right)\right]^{p} \rho d \phi d \rho<+\infty .
$$

Here we use the fact that there is a constant $c$ such that $\rho \leqslant c(1-|z|)$ (this follows, for example, by applying the law of sines to the triangle with vertices at $\zeta, z$, and the point on $C_{1}(0)$ intersected by the radius from 0 through $z$ ).

The key step in the proof is to subdivide $S_{\alpha}(\zeta)$ into countably many annular sectors by inserting arcs of radius $\rho=\rho_{\alpha} / 2^{n}(n=1,2, \ldots)$. Let us use the notation $A\left(\rho^{\prime}, \rho^{\prime \prime}\right)$ for the open annular sector with inner radius $\rho^{\prime}$ and outer radius $\rho^{\prime \prime}$, i.e.,

$$
A\left(\rho^{\prime}, \rho^{\prime \prime}\right)=\left\{\rho e^{i \phi}: \rho^{\prime}<\rho<\rho^{\prime \prime},-\alpha<\phi<\alpha\right\},
$$

and write $\Omega$ for the annular sector $A\left(\rho_{\alpha} / 4,2 \rho_{\alpha}\right)$.

For each $n$ we map $A\left(2^{-n-2} \rho_{\alpha}, 2^{-n+1} \rho_{\alpha}\right)$ conformally onto $\Omega$ by means of a dilation having scale factor $2^{n}$, so that the point $\rho e^{i \phi}$ goes over into the point $t e^{i \phi}$ with

$$
t=2^{n} \rho .
$$

The image of $U$ confined to $A\left(2^{-n-2} \rho_{\alpha}, 2^{-n+1} \rho_{\alpha}\right)$ is thus a superharmonic function $u_{n}$ on $\Omega$ such that

$$
u_{n}\left(t e^{i \phi}\right)=U\left(\rho e^{i \phi}\right),
$$

and it is readily seen that the mass distribution for $u_{n}$ is given by a density function $\lambda_{n}$ satisfying

$$
\lambda_{n}\left(t e^{i \phi}\right)=2^{-2 n} \lambda\left(\rho e^{i \phi}\right) .
$$

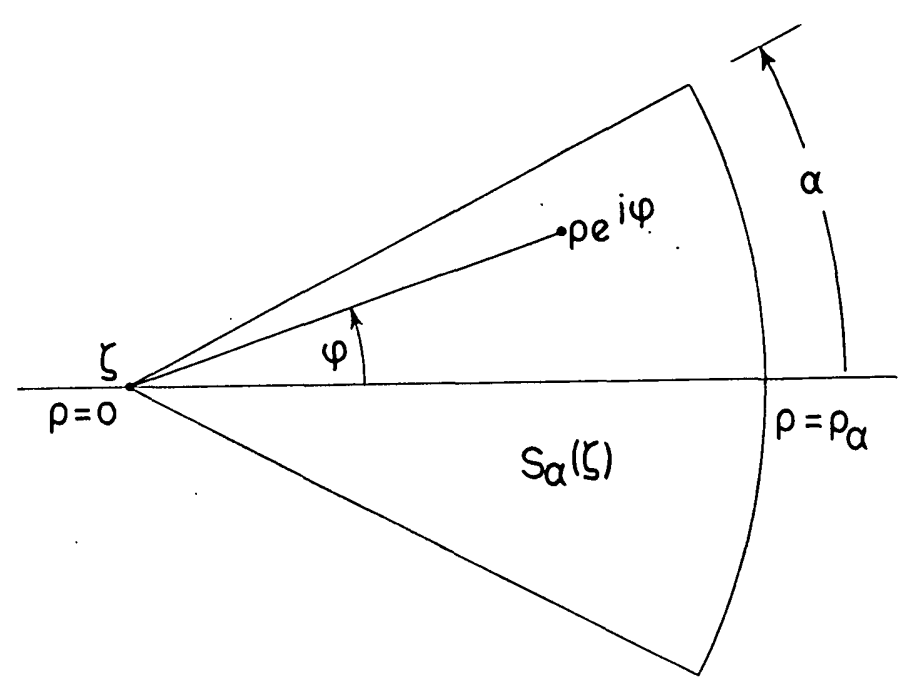

Fig. 2 
This leads at once to the following estimate for the $L^{p}$ norm of $\lambda_{n}$ over $\Omega$.

$$
\begin{aligned}
\left(\left\|\lambda_{n}\right\|_{p}\right)^{p} & =\int_{\rho_{\alpha} / 4}^{2 \rho_{\alpha}} \int_{-\alpha}^{\alpha}\left[\lambda_{n}\left(t e^{i \phi}\right)\right]^{p} t d \phi d t \\
& =\int_{2^{-n-2} \rho_{\alpha}}^{2^{-n+1}} \int_{-\alpha}^{\alpha}\left(2^{-n}\right)^{2(p-1)}\left[\lambda\left(\rho e^{i \phi}\right)\right]^{p} \rho d \phi d \rho \\
& \leqslant\left(\frac{4}{\rho_{\alpha}}\right)^{2(p-1)} \int_{0}^{\rho_{\alpha}} \int_{-\alpha}^{\alpha} \rho^{2(p-1)}\left[\lambda\left(\rho e^{i \phi}\right)\right]^{p} \rho d \phi d \rho .
\end{aligned}
$$

It is thus apparent from (3.5) that $\left\{\lambda_{n}\right\}$ is bounded in $L^{p}$ norm over $\Omega$. To show that $\left\{u_{n}\right\}$ is bounded in $L^{1}$ norm, we appeal to Proposition $\mathrm{D}$, which yields

$$
\begin{aligned}
\left\|u_{n}\right\|_{1} & =\int_{\rho_{\alpha} / 4}^{2 \rho_{\alpha}} \int_{-\alpha}^{\alpha} u_{n}\left(t e^{i \phi}\right) t d \phi d t \\
& =2^{2 n} \int_{2^{-n-2} \rho_{\alpha}}^{2^{-n+1} \rho_{\alpha}} \int_{-\alpha}^{\alpha} U\left(\rho e^{i \phi}\right) \rho d \phi d \rho \leqslant 4 K \rho_{\alpha}{ }^{2} .
\end{aligned}
$$

We are now in a position to apply Proposition C, and for this we first observe from (3.4) that $u_{n} \rightarrow 0$ on a dense subset of $\Omega$ consisting of almost all radial segments. Hence, $u_{n} \rightarrow 0$ uniformly on compact subsets of $\Omega$. For any given $\beta(0<\beta<\pi / 2)$ we can assume that $\alpha$ has been chosen larger than $\beta$. The convergence of $\left\{u_{n}\right\}$ to 0 is then uniform on the compact sector determined by the inequalitios $\rho_{\alpha} / 2 \leqslant t \leqslant \rho_{\alpha}$ and $-\beta \leqslant \phi \leqslant \beta$. This implies that $U(z) \rightarrow 0$ as $z \rightarrow \zeta$ within the Stolz domain $S_{\beta}(\zeta)$, completing the proof.

\section{Proof of Theorem 1}

It is now an easy matter to verify that the condition (1.4) guarantees the existence of finite non-tangential limits at almost all points of $C_{1}(0)$. In fact, fixing $p>1$, we have

$$
\left|\lambda\left(r e^{i \theta}\right)\right|^{p-1} \leqslant \frac{M^{p-1}}{(1-r)^{2(p-1)}} \quad(\delta<r<1)
$$

and hence

$$
\int_{\delta}^{1} \int_{0}^{2 \pi}(1-r)^{2 p-1}\left|\lambda\left(r e^{i \theta}\right)\right|^{p} r d \theta d r \leqslant M^{p-1} \int_{\delta}^{1} \int_{0}^{2 \pi}(1-r)\left|\lambda\left(r e^{i \theta}\right)\right| r d \theta d r .
$$

Since the mass distribution with density $\lambda$ necessarily admits a Green's potential, the inequality in (3.1) ensures that the right-hand integral here is finite, and there remains only to apply Theorem 2.

To prove Theorem 1 in its entirety, we are still obliged to show that the exponent 2 in (1.4) is best possible, i.e., that 2 cannot be replaced by any larger exponent. This will be done by establishing the following property. 
Lemma. For every $\epsilon>0$ there exists a positive mass distribution $m$ on $N_{1}(0)$ such that

(1) $m$ is given by a density function $\lambda$ satisfying

$$
\lim _{r \rightarrow 1} \sup \left[(1-r)^{2+\epsilon} \lambda\left(r e^{i \theta}\right)\right] \leqslant 1
$$

uniformly in $\theta$, and

(2) $m$ admits a Green's potential $U$ satisfying

$$
\limsup _{\substack{z \rightarrow \zeta \\ z \in S_{\alpha}^{(\zeta)}}} U(z) \geqslant \frac{\pi}{2} \epsilon
$$

for all $\zeta$ on $C_{1}(0)$ and all Stolz domains $S_{\alpha}(\zeta)$.

From (1) we see that if the exponent 2 in (1.4) were increased to $2+\epsilon$, the hypothesis of Theorem 1 would be fulfilled by the subharmonic function $u=-U$. On the other hand, (2) shows that $U$ can admit non-tangential limits only at those points $\zeta$ of $C_{1}(0)$ where the radial upper limits of $U$ are positive. Since we know that such points form a set of angular measure zero, it is plain that Theorem 1 would no longer hold with the exponent 2 replaced by $2+\epsilon$.

In order to prove the lemma, we fix $\epsilon>0$ and construct the mass distribution $m$ by spreading mass uniformly over small disks placed on concentric circles about the origin. Let $\left\{\delta_{n}\right\}$ be any monotone decreasing sequence of numbers $0<\delta_{n}<1$ such that the series

$$
\sum_{n=1}^{\infty}\left|\log \delta_{n}\right|^{-\frac{1}{2}}
$$

converges (for example, $\delta_{n}=\exp \left(-n^{4}\right)$ ), and put

$$
\begin{gathered}
r_{n}=1-\delta_{n}, \\
N_{n}=\left[\text { greatest integer } \leqslant \frac{1}{\delta_{n}}\left|\log \delta_{n}\right|^{\ddagger}\right], \\
a_{n}=\delta_{n}^{1+(\epsilon / 2)}\left|\log \delta_{n}\right|^{-\xi} .
\end{gathered}
$$

For each $n$ there will be inserted $N_{n}$ small disks $\sigma_{n}$ of radius $a_{n}$ with centres equally spaced on $C_{r_{n}}(0)$, and mass will be distributed uniformly on these $N_{n}$ disks so as to have density

$$
\lambda_{n}=\delta_{n}^{-(2+\epsilon)} .
$$

Carrying out this procedure for all $n$ gives rise to the mass distribution $m$.

It is easily verified that every Stolz domain contains infinitely many of the inserted disks. We note also that

$$
\lim _{n \rightarrow \infty} \frac{a_{n}}{\delta_{n}}=0,
$$


and the inequality

$$
(1-r)^{2+\epsilon} \lambda\left(r e^{i \theta}\right) \leqslant\left(1+\frac{a_{n}}{\delta_{n}}\right)^{2+\epsilon}
$$

for $r e^{i \theta}$ on $\sigma_{n}$ yields conclusion (1). That $m$ admits a Green's potential is apparent from the expression

$$
\begin{aligned}
\int_{N_{1}(0)}(1-|z|) d m(z) & \leqslant \sum_{n=1}^{\infty}\left(\delta_{n}+a_{n}\right) \pi a_{n}{ }^{2} \lambda_{n} N_{n} \\
& \leqslant \sum_{n=1}^{\infty} \pi\left(1+\frac{a_{n}}{\delta_{n}}\right)\left|\log \delta_{n}\right|^{-\frac{1}{2}}
\end{aligned}
$$

together with the convergence of the final series.

We proceed now to estimate the Green's potential $U$ of $m$ at the centre $z$ of an arbitrary disk $\sigma_{n}$. This can be done simply by observing that the Green's function $G_{z}(\zeta)$ for $N_{1}(0)$ is given by

$$
G_{z}(\zeta)=\log \left|\frac{1-\zeta z}{z-\zeta}\right| \geqslant \log \frac{1-|z|}{|z-\zeta|}
$$

and evaluating the Green's potential of $m$ confined to the one disk centred at $z$. There results

$$
U(z) \geqslant \int_{0}^{a_{n}} \int_{0}^{2 \pi} \lambda_{n} .\left(\log \frac{\delta_{n}}{\rho}\right) \rho d \phi d \rho \geqslant 2 \pi \lambda_{n} \int_{0}^{a_{n}}\left(\log \delta_{n}-\log \rho\right) \rho d \rho .
$$

An elementary calculation then shows that the right-hand member tends to $\pi \epsilon / 2$ as $n \rightarrow \infty$, establishing (2) and completing the proof.

\section{References}

1. M. G. Arsove, "Normal families of subharmonic functions", Proc. American Math. Soc., 7 (1956), 115-126.

2. J. E. Littlewood, " On functions subharmonic in a circle (II) ", Proc. London Math. Soc. (2), 28 (1928), 383-394.

3. I. I. Privalov, Boundary properties of analytic functions (Moscow, 1950) (in Russian).

4. E. B. Tolsted, "Limiting values of subharmonic functions", Proc. American Math. Soc., 1 (1950), 636-647.

5. - " "Non-tangential limits of subharmonic functions", Proc. London Math. Soc. (3), 7 (1957), 321-333.

University of Washington,

Seattle.

Eidgenössische Technische Hochschule, Zurich. 